\title{
Overexpression of MACC1 mRNA in lung adenocarcinoma is associated with postoperative recurrence
}

\author{
Hidehiko Shimokawa, MD, ${ }^{\text {a }}$ Hidetaka Uramoto, MD, PhD, ${ }^{\mathrm{a}}$ Takamitsu Onitsuka, MD, PhD, ${ }^{\mathrm{a}}$ \\ Gu Chundong, MD, PhD, ${ }^{\mathrm{c}}$ Takeshi Hanagiri, MD, PhD, ${ }^{\mathrm{a}}$ Tsunehiro Oyama, MD, PhD, ${ }^{\mathrm{b}}$ and \\ Kosei Yasumoto, $\mathrm{MD}, \mathrm{PhD}^{\mathrm{a}}$
}

Objective: The purpose of this study was to clarify the role and clinical significance of metastasis associated in colon cancer 1 in resected stage I non-small cell lung cancers.

\begin{abstract}
Methods: Tumor specimens were collected from 146 consecutive patients who underwent a complete resection for stage I lung adenocarcinoma from 1998 to 2007 at the University of Occupational and Environmental Health. We analyzed the expression of metastasis associated in colon cancer 1 mRNA of primary lung adenocarcinomas by real-time reverse transcriptase-polymerase chain reaction.
\end{abstract}

Results: The average postoperative observation period was 49.4 months. Thirteen (8.9\%) of 146 patients had recurrences after surgery. Overexpression of metastasis associated in colon cancer 1 mRNA was identified in 62 patients $(42.5 \%)$. Metastasis associated in colon cancer 1 was overexpressed in $9(69.2 \%)$ of 13 patients and 53 $(39.9 \%)$ of 133 patients with and without recurrence, respectively $(P=.004)$. The median metastasis associated in colon cancer 1 copy number was 3.0 and 1.4 in patients with and without tumor recurrence, respectively. Metastasis associated in colon cancer 1 overexpression was associated with poorer disease-free survival according to the survival analysis $(P=.033)$.

Conclusions: Metastasis associated in colon cancer 1 gene overexpression may be a useful marker for predicting postoperative recurrence in patients with lung adenocarcinoma after surgery. (J Thorac Cardiovasc Surg 2011;141:895-8)

Earn CME credits at

http://cme.ctsnetjournals.org

The benefits of adjuvant chemotherapy have been primarily demonstrated using cisplatin-based chemotherapy. ${ }^{1}$ However, the 5-year survival of patients with resected stage IB non-small cell lung cancers is up to $74 \%$ without adjuvant chemotherapy, suggesting that not all patients require chemotherapy after a complete resection. ${ }^{2}$ Therefore, it is necessary to identify the patients who will benefit the most from postoperative adjuvant chemotherapy to precisely select those who will require additional treatment

\footnotetext{
From the Second Department of Surgery, ${ }^{\mathrm{a}}$ Department of Environmental Health, ${ }^{\mathrm{b}}$ School of Medicine, University of Occupational and Environmental Health, Kitakyushu, Japan; and Department of Thoracic Surgery, ${ }^{\mathrm{c}}$ First Affiliated Hospital of Dalian Medical University, Dalian, China.

This work was supported in part by Grants-in-Aid for Scientific Research from the Ministry of Education, Culture, Sports, Science and Technology, Japan.

Disclosures: Authors have nothing to disclose with regard to commercial support.

Received for publication April 11, 2010; revisions received Aug 27, 2010; accepted for publication Sept 10, 2010; available ahead of print Nov 22, 2010.

Address for reprints: Hidetaka Uramoto, MD, $\mathrm{PhD}$, Second Department of Surgery, School of Medicine, University of Occupational and Environmental Health, 1-1 Iseigaoka, Yahatanishi-ku, Kitakyushu 807-8555, Japan (E-mail: hidetaka@med. uoeh-u.ac.jp).

0022-5223/\$36.00

Copyright (C) 2011 by The American Association for Thoracic Surgery

doi:10.1016/j.jtcvs.2010.09.044
}

and to prevent adverse events in those who do not require treatment. Therefore, it is important to evaluate the biological and molecular characteristics of non-small cell lung cancers to identify the factors related to recurrence after surgery. However, no useful markers to predict clinical recurrence are currently known.

The metastasis associated in colon cancer 1 (MACC1) gene was identified by differential display reverse transcriptase-polymerase chain reaction (PCR) by analyzing the colon mucosa, primary tumors, and metastatic lesions of patients with colorectal cancers (CRCs). ${ }^{3}$ MACC1 expression was reported to be a predictor of tumor growth, invasion, and metastasis, and to predict tumor recurrence in colon cancer. ${ }^{4}$ Lung adenocarcinomas are similar to CRCs in regard to histologic type and carcinogenesis. We therefore hypothesized that MACC1 may be a useful prognostic indicator of tumor recurrence in patients after lung adenocarcinoma resection. The purpose of the current study was to clarify the role of MACC1 in lung adenocarcinomas.

\section{MATERIALS AND METHODS \\ Patients and Clinical Features}

Tumor samples were obtained from 494 consecutive patients with primary lung adenocarcinomas who had undergone surgical resection between 1998 and 2007 at the University of Occupational and Environmental Health. Fifty-six patients had an incomplete resection. The tumor samples from 57 patients were too small to extract sufficient levels of DNA for the analyses. A total of 108 patients had overlapping other organs or multiple lung cancers, and 13 patients died of other causes without recurrence. 


\section{Abbreviations and Acronyms \\ $\mathrm{CRC}=$ colorectal cancer \\ DFS $=$ disease-free survival \\ MACC1 $=$ metastasis associated in colon cancer 1 \\ $\mathrm{PCR}=$ polymerase chain reaction}

Fifty-four patients had pathologic diagnoses of stage II to IV tumors. As a result, 348 patients were excluded from the analyses. A total of $146 \mathrm{pa}-$ tients were included in the present series. All patients were Japanese. There were 77 male subjects and 69 female subjects in the current series, with a mean age of 68.3 years (range, $40-91$ years). The tumor stage was classified according to the new TNM (7th edition) classification for lung cancer. $^{5}$ According to the pathologic stage, 116 patients had stage IA tumors, including 71 with stage T1a, 45 with stage T1b, and 30 with stage IB (T2a). The operative procedures included a lobectomy in 133 patients, segmentectomy partial resection in 5 patients, partial resection in 5 patients, lobectomy plus partial resection in 2 patients, and lobectomy plus segmentectomy in 1 patient. No patients had received chemotherapy or radiotherapy before the resection. Nine patients $(6.2 \%)$ had received adjuvant chemotherapy (7 patients received carboplatin plus paclitaxel, 1 patient received carboplatin plus gemcitabine, and 1 patient received tegafur-uracil). The average postoperative observation period was 51.1 months, ranging from 1.8 to 126.5 months. Thirteen $(8.9 \%)$ of 146 patients had recurrences after surgery.

We investigated MACC1 overexpression, the relationship between MACC1 overexpression and clinicopathologic factors, and the recurrence rate by MACC1 discrimination after surgery in patients with lung adenocarcinoma. The institutional review board's approved informed consent for the use of the tumor specimens, data collection, and analysis was obtained from the patients or their legal guardian(s). Patients were followed up every month within the first postoperative year and at approximately 2- to 4-month intervals thereafter. Evaluations included physical examination, chest roentgenography, blood chemistry analysis, and measurement of

TABLE 1. Expression of MACC1 mRNA in normal tissue

\begin{tabular}{lc}
\hline \multicolumn{1}{c}{ Organ } & Expression \\
\hline Adrenal gland & 0.0055 \\
Bone marrow & 0.1329 \\
Bronchus & 0.3192 \\
Colon & 0.0502 \\
Fetal brain & 0.0045 \\
Fetal liver & 0.0161 \\
Heart & 0.0651 \\
Kidney & 1.8185 \\
Liver & 0.0433 \\
Lung & 1.0024 \\
Placenta & 0.0854 \\
Prostate & 0.6149 \\
Salivary gland & 0.9055 \\
Skeletal muscle & 0.0024 \\
Spinal cord & 0.0049 \\
Testis & 0.1441 \\
Thymus & 0.0295 \\
Thyroid gland & 0.6212 \\
Uterus & 0.0049 \\
Whole brain & 0.0079 \\
\hline
\end{tabular}

MACC1, Metastasis associated in colon cancer 1.
TABLE 2. Relationships between MACC1 overexpression and clinicopathologic characteristics

\begin{tabular}{llccc}
\hline & & \multicolumn{2}{c}{ MACC1 overexpression } \\
\cline { 3 - 5 } Variables & Category & $\begin{array}{c}\text { No. of patients } \\
\mathbf{n}=\mathbf{1 4 6}\end{array}$ & $\begin{array}{c}\text { Positive (\%) } \\
\text { (42 (42.5) }\end{array}$ & $\begin{array}{c}\text { Negative } \\
\mathbf{8 4}\end{array}$ \\
\hline \multirow{2}{*}{ Gender } & Male & 77 & $39(50.6)$ & 38 \\
& Female & 69 & $23(33.3)$ & 46 \\
Age (y) & $\leq 69$ & 77 & $33(42.9)$ & 44 \\
& $>69$ & 69 & $29(42.0)$ & 40 \\
pT & T1 & 116 & $49(42.2)$ & 67 \\
& T2-4 & 30 & $13(43.3)$ & 17 \\
\hline
\end{tabular}

$p T$, Pathologic T status; $M A C C l$, metastasis associated in colon cancer 1.

tumor markers (eg, carcinoembryonic antigen, CYFRA, and squamous cell carcinoma). Chest and abdominal computed tomography, brain magnetic resonance imaging, and a bone scintiscan were performed every 6 months for 3 years after surgery. Additional examinations were performed if any symptoms or signs of recurrence were detected.

\section{Detection of MACC1 Overexpression}

MACC1 overexpression was analyzed in all samples by quantitative real-time PCR, performed on a StepOnePlus Real-Time PCR System (Applied Biosystems, Foster City, Calif) using a Fast SYBR Green Master Mix (Applied Biosystems). Gene expression was quantified by comparing the levels of the target gene with the levels of beta actin as an internal control. Quantification was based on a standard curve generated from human normal genomic DNA by previously described methods. ${ }^{6}$ The relative MACC1 copy number was also normalized to human genomic DNA. The change in the copy number of the MACC1 gene relative to actin and the calibrator DNA were determined by the following formula: (tumorMACC1/tumor-beta actin)/(control-MACC1/control-beta actin). PCR for each primer set was performed in triplicate, and the means were reported. The conditions for the quantitative PCR reactions were as follows: 1 cycle of $95^{\circ} \mathrm{C}$ for 20 seconds, 40 cycles of $95^{\circ} \mathrm{C}$ for 3 seconds, and $60^{\circ} \mathrm{C}$ for 30 seconds. At the end of the PCR, the samples were subjected to a melting analysis to confirm the specificity of the amplicon. The primer sequences used in the present study for the MACC1 gene were as follows: forward, TTCTTTTGATTCCTCCGGTGA, and reverse, ACTCTGATGGGCATGTGCTG. ${ }^{3}$ A commercially available panel of total RNA (Clontech Laboratories Inc, Palo Alto, Calif) obtained from 20 normal tissues was used. We assigned a score of 1.0 as the MACC 1 gene copy number present in normal lung tissues.

\section{Statistical Analysis}

Statistical significance was evaluated using the chi-square or Fisher's exact test. The odds ratio and $95 \%$ confidence interval were calculated for each variable. The Kaplan-Meier method was used to estimate the probability of survival, and survival differences were analyzed by the log-rank test. A multivariate analysis was then performed according to

TABLE 3. Recurrent sites of tumors

\begin{tabular}{llc}
\hline Hematogenous $(\mathbf{n}=\mathbf{9} *)$ & \multicolumn{1}{c}{ Site } & No. $\dagger$ \\
\hline & Brain & 1 \\
& Lung & 7 \\
& Adrenal & 1 \\
& Skin & 1 \\
& Liver & 1 \\
Locoregional $(n=5)$ & Lymph node & 3 \\
& Pleural dissemination & 2 \\
\hline
\end{tabular}

*One patient had recurrent tumors in the brain and adrenal gland, and 1 patient had recurrent tumors in the lung and liver. $\dagger$ The number of recurrent sites overlapped. 
TABLE 4. Relationships between MACC1 overexpression and recurrence

\begin{tabular}{lcc}
\hline & \multicolumn{2}{c}{ MACC1 overexpression } \\
\cline { 2 - 3 } \multicolumn{1}{c}{ Variables } & Positive (\%) 62 (42.5) & Negative 84 \\
\hline Cases with recurrence & $9(69.2)$ & 4 \\
Cases without recurrence & $53(39.9)$ & 80 \\
\hline
\end{tabular}

MACCl, Metastasis associated in colon cancer 1.

Cox's proportional hazards model. Data were analyzed using the StatView software package (Abacus Concepts, Inc, Berkeley, CA).

\section{RESULTS}

\section{Detection of MACC1 Overexpression}

The maximum MACC1 overexpression in normal tissue was 1.82 in the kidney, compared with the value of 1.0 defined for normal lung tissue (Table 1). Therefore, we assigned a cutoff value of 2.0 for MACC1 overexpression, which was defined as 2 or more copies. Furthermore, 5 (71.4\%) of 7 metastatic lung tumors from CRCs were confirmed to have overexpressed MACC1 expression (data not shown). MACC1 overexpression was present in the tumors of 93 patients $(46.5 \%)$. There was no significant association of MACC1 overexpression with the clinical factors (Table 2).

\section{Relationships Between MACC1 Overexpression and Recurrence}

The majority of the sites of tumor recurrence were hematogenous metastases (Table 3). MACC1 was overexpressed in $9(69.2 \%)$ of 13 patients and $53(39.9 \%)$ of 133 patients with and without recurrence, respectively $(P=.004)$ (Table 4). The median MACC1 copy number was 3.0 in patients with tumor recurrence and 1.4 in patients without recurrence.

\section{Prognosis of Patients According to Molecular Parameters}

The 5-year disease-free survival (DFS) for those with negative and positive MACC1 overexpression was $97.4 \%$

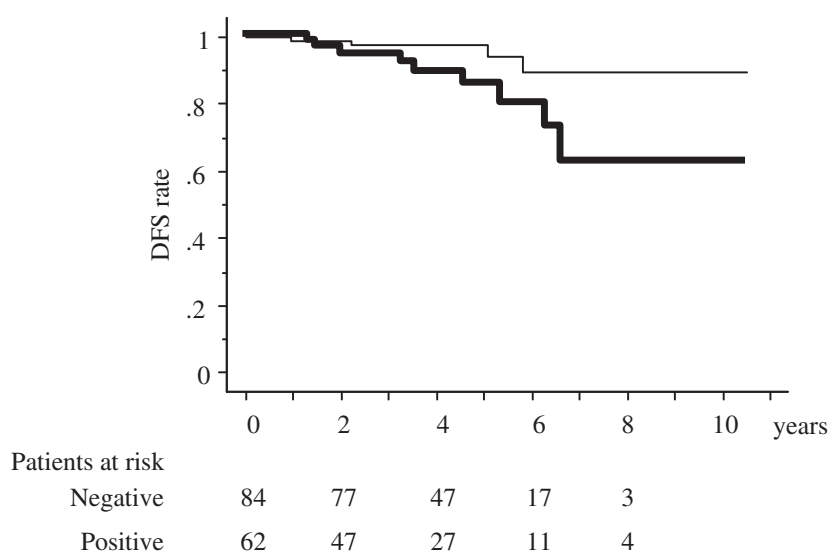

FIGURE 1. Kaplan-Meier DFS curves stratified by MACC1 expression. The positive (heavy lines) and negative (narrow lines) expression of MACC1. MACC1, Metastasis associated in colon cancer 1.
TABLE 5. Univariate analysis using a proportional hazard model for disease-free survival

\begin{tabular}{|c|c|c|c|c|c|}
\hline \multirow[b]{2}{*}{ Variables } & \multicolumn{2}{|c|}{ Characteristics } & \multirow{2}{*}{$\begin{array}{l}\text { Hazard } \\
\text { ratio }\end{array}$} & \multirow[b]{2}{*}{$95 \% \mathrm{CI}$} & \multirow{2}{*}{$\begin{array}{c}P \\
\text { value }\end{array}$} \\
\hline & Unfavorable & Favorable & & & \\
\hline Gender & Male & Female & 1.767 & $0.543-5.747$ & .334 \\
\hline Age (y) & $\leq 69$ & $>69$ & 0.963 & $0.323-2.876$ & .947 \\
\hline $\mathrm{T}$ status & $\mathrm{T} 2 \mathrm{a}$ & $\mathrm{T} 1$ & 2.695 & $0.882-8.264$ & .082 \\
\hline $\begin{array}{l}\text { MACC } 1 \\
\text { overexpression }\end{array}$ & Positive & Negative & 3.356 & $1.031-10.870$ & .044 \\
\hline
\end{tabular}

and $85.4 \%$, respectively $(P=.033$ ) (Figure 1$)$. MACC1 overexpression was associated with poorer DFS according to the univariate survival analysis $(P=.044)$ (Table 5). We also divided the tertile classification and analysis by the division of the approximate number of the patients: less than $1.0(\mathrm{n}=39)$, more than 1.0 but less than 3.0 $(\mathrm{n}=50)$, and more than $3.0(\mathrm{n}=57)$. The 5-year DFS of the less than 1.0, more than 1.0 but less than 3.0, and more than 3.0, for MACC1 overexpression, was $97.4 \%$, $95.9 \%$, and $86.2 \%$, respectively. Multivariate analysis showed a $P$ value of .044 when divided by the number of specimens with positive or negative MACC1 expression. The $P$ value was .621 when divided by the number with more than 1.0 or less than 1.0 , and the $P$ value was .201 when divided by the number more than 3.0 or less than 3.0 (Table 6).

\section{DISCUSSION}

Clinical findings and current tissue-based molecular markers are insufficient for the early identification of individuals at high risk for metastasis. ${ }^{7}$ Therefore, novel strategies for prevention of metastasis are urgently required to improve the prognosis and quality of life for patients with cancer. MACC1 was recently identified as an important prognostic factor of metastatic disease in CRC. ${ }^{3}$ In addition, MACC1 is located on chromosome 7p21.1 and regulates tissue growth via the hepatocyte growth factor/MET signaling pathway. ${ }^{4}$ Hepatocyte growth factor/MET overexpression is an important predictor of the response of patients with lung adenocarcinoma treated with an epidermal growth factor receptor-tyrosine kinase inhibitor. ${ }^{8}$ Notably, polysomy of chromosome 7 is a common finding in both lung cancers and CRC tumors. ${ }^{9-11}$ Therefore, we hypothesized that the incidence of MACC1 overexpression would be higher in recurrent cases after surgery because these share the same histology and carcinogenesis resembles CRC. If this is the case, then this subgroup of patients with stage I should be selected for adjuvant chemotherapy with priority as higher risk.

The current study is the first to demonstrate the molecular analysis of MACC1 overexpression and correlate this gene to the prognosis of patients with lung adenocarcinoma. The 
TABLE 6. Multivariate analysis using a proportional hazard model for disease-free survival

\begin{tabular}{|c|c|c|c|c|c|}
\hline \multirow[b]{2}{*}{ Variables } & \multicolumn{2}{|c|}{ Characteristics } & \multirow[b]{2}{*}{ Hazard ratio } & \multirow[b]{2}{*}{$95 \% \mathrm{CI}$} & \multirow[b]{2}{*}{$P$ valu } \\
\hline & Unfavorable & Favorable & & & \\
\hline \multicolumn{6}{|l|}{ Model 1} \\
\hline $\mathrm{T}$ status & $\mathrm{T} 2 \mathrm{a}$ & $\mathrm{T} 1$ & 2.741 & $0.886-8.333$ & .081 \\
\hline MACC1 overexpression & Positive & Negative & 3.3675 & $1.034-10.989$ & .044 \\
\hline \multicolumn{6}{|l|}{ Model 2} \\
\hline $\mathrm{T}$ status & $\mathrm{T} 2 \mathrm{a}$ & $\mathrm{T} 1$ & 2.564 & $0.828-7.937$ & .103 \\
\hline MACC1 overexpression & $>1.0$ & $<1.0$ & 1.470 & $0.319-6.770$ & .621 \\
\hline \multicolumn{6}{|l|}{ Model 3} \\
\hline $\mathrm{T}$ status & $\mathrm{T} 2 \mathrm{a}$ & $\mathrm{T} 1$ & 2.725 & $0.889-8.333$ & .079 \\
\hline MACC1 overexpression & $>3.0$ & $<3.0$ & 2.044 & $0.684-6.107$ & .201 \\
\hline
\end{tabular}

CI, Confidence interval; $M A C C 1$, metastasis associated in colon cancer 1.

results show that the incidence of MACC1 overexpression was significantly higher in recurrent cases than in nonrecurrent cases. Furthermore, MACC1 overexpression was associated with poorer DFS (Figure 1). Stein and colleagues $^{3}$ reported a 5-year survival of $80 \%$ for patients with low MACC1-expressing tumors, but only $15 \%$ for individuals with high MACC1-expressing primary tumors, which was consistent with our results. The biological findings in lung adenocarcinoma suggested a sequential progression from localized bronchioloalveolar carcinomas during the course of pulmonary adenocarcinogenesis. ${ }^{12}$ These carcinogenesis events seem similar to the transition from adenoma to carcinoma in CRCs. ${ }^{4}$ However, the reason for the difference in predictability by MACC 1 between lung adenocarcinoma and CRC still remains to be elucidated. This finding suggests that MACC1 overexpression may be a suitable biomarker to identify those candidates who would benefit most from adjuvant chemotherapy in stage I adenocarcinoma after complete resection.

\section{CONCLUSIONS}

MACC1 overexpression has the potential to be a predictor of postoperative tumor recurrence in lung adenocarcinoma after complete resection. However, the present study had 2 limitations: (1) This was a retrospective study performed at a single institution, and (2) not all models by multivariate analysis demonstrated that MACC1 overexpression was independently associated with poor patient survival. To overcome these limitations, further exploratory studies of biomarkers are required to predict tumor recurrence after pulmonary resection.

The authors thank Misako Fukumoto for valuable technical assistance and Yoshihisa Fujino, MD, MPH, Department of Preven- tive Medicine and Community Health, University of Occupational and Environmental Health for statistical advice.

\section{References}

1. Pignon JP, Tribodet H, Scagliotti GV, Douillard JY, Shepherd FA, Stephens RJ, et al. Lung adjuvant cisplatin evaluation: a pooled analysis by the LACE Collaborative Group. J Clin Oncol. 2008;26:3552-9.

2. Kato H, Ichinose Y, Ohta M, Hata E, Tsubota N, Tada H, et al. Japan Lung Cancer Research Group on Postsurgical Adjuvant Chemotherapy. A randomized trial of adjuvant chemotherapy with uracil-tegafur for adenocarcinoma of the lung. N Engl J Med. 2004;350:1713-21.

3. Stein U, Walther W, Arlt F, Schwabe H, Smith J, Fichtner I, et al. MACC1, a newly identified key regulator of HGF-MET signaling, predicts colon cancer metastasis. Nat Med. 2009;15:59-67.

4. Arlt F, Stein U. Colon cancer metastasis: MACC1 and Met as metastatic pacemakers. Int J Biochem Cell Biol. 2009;41:2356-9.

5. Vallières E, Shepherd FA, Crowley J, Van Houtte P, Postmus PE, Carney D, et al., International Association for the Study of Lung Cancer International Staging Committee and Participating Institutions. The IASLC Lung Cancer Staging Project: proposals regarding the relevance of TNM in the pathologic staging of small cell lung cancer in the forthcoming (seventh) edition of the TNM classification for lung cancer. J Thorac Oncol. 2009;4:1049-59.

6. Onitsuka T, Uramoto H, Nose N, Takenoyama M, Hanagiri T, Sugio K, et al. Acquired resistance to gefitinib: the contribution of mechanisms other than the T790M, MET, and HGF status. Lung Cancer. 2010;68:198-203.

7. Duffy MJ, van Dalen A, Haglund C, Hansson L, Holinski-Feder E, Klapdor R, et al. Tumour markers in colorectal cancer: European Group on Tumour Markers (EGTM) guidelines for clinical use. Eur J Cancer. 2007;43:1348-60.

8. Onitsuka T, Uramoto H, Ono K, Takenoyama M, Hanagiri T, Oyama T, et al. Comprehensive molecular analyses of lung adenocarcinoma with regard to the epidermal growth factor receptor, K-ras, MET, and hepatocyte growth factor status. J Thorac Oncol. 2010;5:591-6.

9. Weidner KM, Arakaki N, Hartmann G, Vandekerckhove J, Weingart S, Rieder H, et al. Evidence for the identity of human scatter factor and human hepatocyte growth factor. Proc Natl Acad Sci U S A. 1991;88:7001-5.

10. Kitada K, Yamasaki T. The complicated copy number alterations in chromosome 7 of a lung cancer cell line is explained by a model based on repeated breakagefusion-bridge cycles. Cancer Genet Cytogenet. 2008;185:11-9.

11. Casorzo L, Corigliano M, Ferrero P, Venesio T, Risio M. Evaluation of 7q31 region improves the accuracy of EGFR FISH assay in non small cell lung cancer. Diagn Pathol. 2009;4:36.

12. Aoyagi Y, Yokose T, Minami Y, Ochiai A, Iijima T, Morishita Y, et al. Accumulation of losses of heterozygosity and multistep carcinogenesis in pulmonary adenocarcinoma. Cancer Res. 2001;61:7950-4. 